\title{
Anticonvulsant drug actions on neurons in cell culture
}

\author{
R. L. Macdonald \\ Department of Neurology, University of Michigan Medical Center, Ann Arbor, Michigan, \\ U.S.A.
}

Summary. Two actions of clinically used antiepileptic drugs have been studied using mouse neurons in primary dissociated cell culture. The antiepileptic drugs phenytoin, carbamazepine and valproic acid were demonstrated to limit sustained high frequency repetitive firing of action potentials at free serum concentratons that are achieved in patients being treated for epilepsy. Furthermore, an active metabolite of carbamazepine also limited sustained high frequency repetitive firing while inactive metabolites of phenytoin and carbamazepine did not limit sustained high frequency repetitive firing. Phenobarbital and clinically used benzodiazepines limited sustained high frequency repetitive firing of action potentials, but only at concentrations achieved during the treatment of generalized tonic-clonic status epilepticus. Ethosuximide did not limit sustained high frequency repetitive firing even at concentrations four times those achieved in the serum of patients treated for generalized absence seizures. Phenobarbital and clinically used benzodiazepines enhanced postsynaptic GABA responses at concentrations achieved free in the serum during treatment of generalized tonic-clonic or generalized absence seizures. However, phenytoin, carbamazepine, valproic acid and ethosuximide did not modify postsynaptic GABA responses at therapeutic free serum concentrations. These results suggest that the ability of antiepileptic drugs to block generalized tonicclonic seizures and generalized tonic-clonic status epilepticus may be related to their ability to block high frequency repetitive firing of neurons. The mechanism underlying blockade of myoclonic seizures may be related to the ability of antiepileptic drugs to enhance GABAergic synaptic transmission. The mechanism underlying management of generalized absence seizures remains unclear.

Keywords: Antiepileptic drugs; action potentials; sodium channels; GABA.

\section{Introduction}

Antiepileptic drugs have been demonstrated to be of use in the management of patients with various types of epilepsy. Patients can be successfully managed for generalized tonic-clonic, generalized absence and myoclonic seizures with 
a number of primary antiepileptic drugs (Woodbury et al., 1982). The primary drugs that are currently in use are phenytoin, carbamazepine, primidone, phenobarbital, clonazepam, valproic acid and ethosuximide. Selection of antiepileptic drug is determined by the type of seizure disorder that has been diagnosed. For example, in patients with primary generalized tonic-clonic seizures, the drug of choice is phenytoin or carbamazepine, although valproic acid has been shown to be as effective as the two first-line drugs. In contrast, myoclonic seizures are treated primarily by valproic acid or benzodiazepines such as clonazepam. In the therapy of generalized absence seizures, ethosuximide is the drug of choice, but for generalized absence seizures in combination with other seizure types, valproic acid is more effective. Benzodiazepines such as clonazepam have been shown also to be effective, but their use is often limited by the development of tolerance.

The major antiepileptic drugs also have a different spectrum of action against seizures induced in experimental animals (Krall et al., 1978). Two primary experimental seizures have been used to assess the effectivenes of these drugs. Maximal electroshock seizures can be blocked at nontoxic doses by phenytoin, carbamazepine, primidone, phenobarbital and valproic acid. Clonazepam will block maximal electroshock seizures, but only at toxic concentrations. Ethosuximide is relatively ineffective against this experimental seizure type. Subcutaneous pentylenetetrazol produces primarily clonic seizures in experimental animals. These seizures can be blocked by phenobarbital, clonazepam, valproic acid and ethosuximide. Phenytoin, carbamazepine and primidone are relatively ineffective against this seizure type.

The basic mechanisms underlying the selectivity of antiepileptic drugs are uncertain. Data obtained from the clinical use of antiepileptic drugs and from antiepileptic drug selectivity in experiment animals suggest that antiepileptic drugs may have multiple mechanisms of action. Recently, two actions of antiepileptic drugs have been shown to be potential antiepileptic mechanisms; reduction of sustained high frequency repetitive firing and enhancement of GABAergic synaptic transmission (Macdonald, 1983; Macdonald and McLean, 1986). In this article, I will review some data supporting the hypotheses that these two actions are antiepileptic.

\section{Materials and methods}

Intracellular recordings have been made from spinal cord, cortical and hippocampal neurons grown in primary dissociated cell culture. Neurons were taken from the relevant location in the spinal cord or central nervous system, mechanically disrupted, dissociated and plated as a single cell suspension on collagen-coated $35 \mathrm{~mm}$ plastic dishes. While culture conditions varied somewhat depending upon the region of the nervous system that was placed in culture, cultures were grown for 4-12 weeks prior to intracellular recording. For specific details of the culture technique, see McLean and Macdonald, 1986.

Intracellular recordings were made from neurons bathed in a physiological salt solution at $37^{\circ} \mathrm{C}$. Cells were accepted only if their resting membrane potentials exceeded $-50 \mathrm{mV}$. From resting membrane potential a series of depolarizing current pulses of variable du- 
rations were applied and the evoked action potentials were photographed. Under control conditions, spinal cord and cortical neurons respond to progessively increasing depolarizing pulses with an increasing number and frequency of action potentials. Within $5 \mathrm{mV}$ of obtaining threshold for the initial action potentials, a steady rate of action potential firing can be evoked. With increasing depolarizing pulses above threshold, the rate of firing increases. To asses the effect of antiepileptic drugs, recordings were obtained from a series of spinal cord, cortical or hippocampal neurons. Cultures were discarded if sustained repetitive firing could not be elicited in the control neurons. An antiepileptic drug at an appropriate concentration was added to the bath and mixed. Different neurons were then recorded from and the results on repetitive firing were compared to those obtained from neurons in control medium.

To study the effect of antiepileptic drugs on GABA responses, iontophoretic pipettes filled with GABA (0.5 M, pH 3.5) positioned within $2 \mu$ of the surface of a neuron which had been impaled. Positive current pulses (100 to $400 \mathrm{msec}$ ) were applied to the iontophoretic pipettes at a rate of $0.2 \mathrm{~Hz}$. Intracellular recordings were made with potassium chloride $(3 \mathrm{M})$ filled micropipettes, and membrane potential was held more negative than $-70 \mathrm{mV}$. Under these conditions, GABA responses were depolarizing since the chloride equilibrium potential is about $-30 \mathrm{mV}$. After a stable GABA response which was less than $10 \mathrm{mV}$ was recorded, a blunt-tipped pipette containing an antiepileptic drug which was dissolved in recording medium was brought up adjacent to the iontophoretic pipette and either pressure applied or allowed to bathe the area by local diffusion. After a stable enhancement or reduction of GABA responses was recorded, the antiepileptic drug-containing pipette was withdrawn and control responses were recovered. All data were discarded if control and recovery GABA responses were not within $5 \%$ of each other.

Selection of antiepileptic drug concentration was guided by information obtained in human studies. Antiepileptic drugs vary in their protein binding in serum. Benzodiazepines such as diazepam are bound to a high degree (97-98\%). In contrast ethosuximide is not protein bound. It is the unbound anticonvulsant drug which equilibrates with cerebrospinal fluid and therefore extracellular space in the nervous system. Therefore, an action of an antiepileptic drug should occur at a concentration which is found in CSF or which is found free in plasma. Accordingly actions of antiepileptic drugs were considered potentially relevant for clinical use if they occurred at concentrations of antiepileptic drugs in free serum or CSF during therapy.

\section{Results}

\section{Reduction of sustained high frequency repetitive firing by antiepileptic drugs}

Intracellular recordings were obtained from spinal cord, cortical and hippocampal neurons in cell culture. When a 400-500 msec depolarizing current was applied through the recording pipette, a volley of action potentials was elicited (Fig. 1, CONT). Increasing the magnitude of the current injection produced increased membrane depolarization associated with volleys of action potentials at higher frequencies. Over a wide range of depolarizing pulses, action potential firing was sustained throughout the pulse. The frequencies of action potential firing approached $500 \mathrm{~Hz}$. Therefore, in control neurons, depolarizing current injections produced sustained high frequency repetitive firing. When sodium valproate (McLean and Macdonald, 1986a), phenytoin (McLean and Macdonald, 1983b) and carbamazepine (McLean and Macdonald, 1983a; 1986b) were bath applied, a different pattern of repetitive firing was observed (Fig. 1). In a dose-dependent fashion, all three antiepileptic drugs limited the ability of 
individual neurons to sustain high frequency repetitive firing. At increasing antiepileptic drug concentration, an increasing percentage of neurons failed to respond to depolarizing current pulses with sustained firing. Instead, these neurons fired a series of action potentials at moderately high frequency with rapidly declining amplitudes and maximal rates of rise. During the depolarizing pulse, action potentials failed. This failure to sustain high frequency repetitive firing was seen over a large range of depolarizing current pulses. While all three antiepileptic drugs limited sustained high frequency repetitive firing, they did not affect the amplitude, duration or maximal rate of rise of the first action potential in each train. Sodium valproate limited sustained repetitive firing at concentrations above $0.6 \mu \mathrm{M}$ and approximately $80 \%$ of neurons showed limitation at $12 \mu \mathrm{M}$. Phenytoin limited sustained high frequency repetitive firing in neurons at concentrations above $0.2 \mu \mathrm{M}$ and limited sustained repetitive firing in $80 \%$ of neurons at $8 \mu \mathrm{M}$. Carbamazepine limited sustained high frequency repetitive firing in neurons above $0.84 \mu \mathrm{M}$ and produced an $80 \%$ reduction in the percentage of neurons firing sustained repetitive firing at $4.2 \mu \mathrm{M}$. Concentrations over which sodium valproate, phenytoin and carbamazepine had their action on sustained repetitive firing were equal to the free serum concentrations achieved in patients treated for generalized tonic-clonic seizures with these antiepileptic drugs.

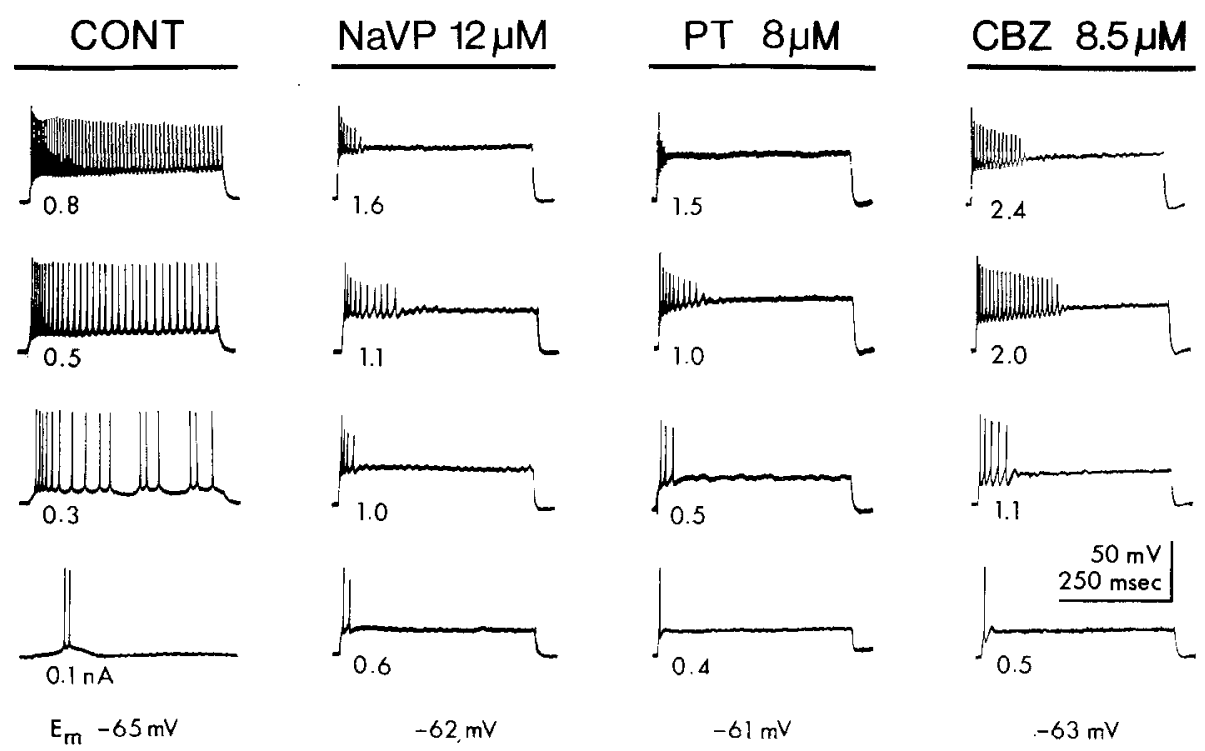

Fig. 1. Valproic acid $(N a V P)$, phenytoin $(P T)$, and carbamazepine $(C B Z)$ limited sustained high frequency repetitive firing. Intracellular recordings were made from spinal cord neurons in cell culture and increasing depolarizing current pulses were applied. Magnitude of the current pulses is shown below each of the traces and the membrane potentials from which the depolarizing pulses were obtained are shown below each column. In control (CONT) medium sustained high frequency repetitive firing was obtained throughout all ranges of depolarization. In the presence of sodium valproate $(12 \mu \mathrm{M})$, phenytoin $(8 \mu \mathrm{M})$, and carbamazepine $(8.5 \mu \mathrm{M})$ limitation of sustained repetitive firing was seen at each of the membrane depolarization (McLean and Macdonald, unpublished) 
Antiepileptic drugs are metabolized in the liver and the resultant metabolites attained levels in the cerebrospinal fluid and in the nervous system. We have studied some of these metabolites to determine whether or not they had similar effects on repetitive firing. Phenytoin is metabolized primarily to the parahydroxylated derivative 5-p-hydroxyphenyl-5-phenyl hydantoin (HPPH). HPPH is not antiepileptic when administered to experimental animals but does not cross the blood brain barrier in large quantities. Therefore it is not certain whether it has an antiepileptic action. However, HPPH had no effect on sustained high frequency repetitive firing at concentrations up to $40 \mu \mathrm{M}$ (McLean and Macdonald, 1983b). Carbamazepine is metabolized to 2 metabolites, carbamazepine epoxide and carbamazepine dihydroxide. Carbamazepine epoxide has been shown to have antiepileptic action in experimental animals while carbamazepine dihydroxide has been shown to ineffective against experimental seizures. Carbamazepine epoxide limited sustained high frequency repetitive firing at concentrations comparable to carbamazepine while the dihydroxide derivative of carbamazepine was not effective at concentrations 10 times those effective for carbamazepine (McLean and Macdonald, 1983a; 1986 b). Valproic acid has many metabolites but one major metabolite is the 2-en-valproic acid (2-en-VPA) (Nau and Löscher, 1982). 2-en-VPA had no effect on sustained high frequency repetitive firing (McLean and Macdonald, 1986 a) at concentrations achieved in experimental animals (Nau and Löscher, 1982). These results suggest that one metabolite of carbamazepine may also contribute to its antiepileptic action but that the metabolites of phenytoin and valproic acid studied may be ineffective.

In contrast to the actions of sodium valproate, phenytoin and carbamazepine, the antiepileptic drugs primidone, phenobarbital, clonazepam and ethosuximide did not limit sustained high frequency repetitive firing a therapeutic free serum concentrations (Skerritt et al., 1984a, b; Macdonald and McLean, 1986; McLean and Macdonald, 1986 a). Primidone, phenobarbital and clonazepam limited sustained high frequency repetitive firing but only at concentrations that exceeded therapeutic free serum concentrations. Concentrations required for primidone and phenobarbital were over $200 \mu \mathrm{M}$ and for clonazepam were over $50 \mathrm{nM}$. These concentrations are achieved in patients being treated for generalized tonic-clonic status epilepticus but are generally not achieved in ambulatory patients. These are usually sedating concentrations of antiepileptic drugs. Ethosuximide did not limit repetitive firing even at concentrations which were supratherapeutic.

The absence of effect of the antiepileptic drugs on resting membrane properties suggested that the antiepileptic drugs were acting directly on sodium channels to limit repetitive firing. In spinal cord and cortical neurons, action potentials are primarily sodium-dependent. The maximal rate of rise of action potentials has been used as an indirect measure of the inward sodium current. In spinal cord neurons, we have demonstrated that the amplitude of action potentials is proportional to the logarithm of the extracelluiar sodium concentrations, thus demonstrating that these action potentials are sodium-dependent. Therefore, to study the effect of antiepileptic drugs on sodium channels, we used the maximal rate of rise of the action potential as an indirect assay of sodium current. 


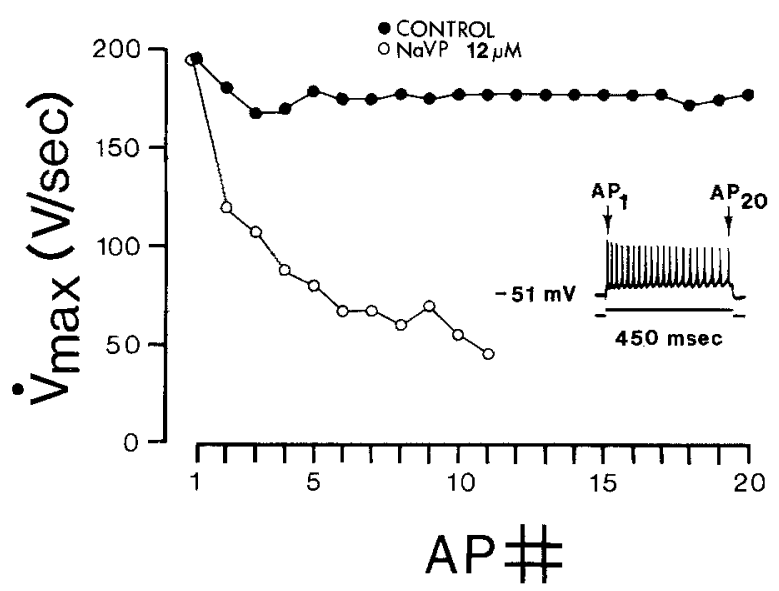

Fig. 2. Limitation of sustained repetitive firing by sodium valproate, phenytoin and carbamazepine was use-dependent. The use-dependent effect of the antiepileptic drugs is shown for NaVP. In control medium, sustained repetitive firing was obtained (control). The maximum rate of rise of individual action potentials $\left(\dot{\mathrm{V}}_{\max }\right)$ is plotted as a function of the action potential number within a train (see insert). In control medium, the initial action potential had the largest $\dot{\mathrm{V}}_{\text {max }}$. Subsequent action potentials had slightly smaller $\dot{\mathrm{V}}_{\max }$ values until a relative steady state was achieved. in NaVP $(12 \mu \mathrm{M})$, the $\dot{\mathrm{V}}_{\max }$ of the initial action potential was identical to that seen in control medium. However, there was a rapid reduction in $\dot{V}_{\max }$ as a function of the number of action potential in the train. In NaVP, only 11 action potentials were evoked and repetitive firing was therefore limited. This reduction in the number of action potentials was associated with this use-dependent reduction of the $\dot{\mathrm{V}}_{\max }$ of the action potentials (from McLean and Macdonald, 1986)

The effect of sodium valproate, phenytoin and carbamazepine varied depending upon the membrane potential of the neuron, i.e., the effect of these antiepileptic drugs on repetitive firing was voltage-dependent. The ability of the antiepileptic drugs to limit repetitive firing was apparent only following membrane depolarization. When neurons were depolarized, a clear reduction in sustained repetitive firing was produced by the antiepileptic drugs. However, following membrane hyperpolarization, neurons could achieve sustained repetitive firing despite the presence of the antiepileptic drug.

The effect of the antiepileptic drugs on sustained repetitive firing was also use- or frequency-dependent (Fig. 2). The antiepileptic drugs did not modify the rate of rise, amplitude or duration of single action potentials evoked over a wide range of voltages. However, with repetitive stimulation or with sustained depolarization producing repetitive firing, there was a progressive reduction in the amplitude and maximal rate of rise of successive action potentials. With repetitive stimulation or sustained depolarization, failure of action potential firing occurred. Thus, to produce the reduction in action potentials duration, rate of rise and amplitude, repeated action potentials had to be evoked.

\section{Enhancement of GABA responses by antiepileptic drugs}

Phenobarbital (Macdonald and Barker, 1979a; Schulz and Macdonald, 1981) and the benzodiazepines diazepam, nitrazepam and clonazepam (Choi et al., 


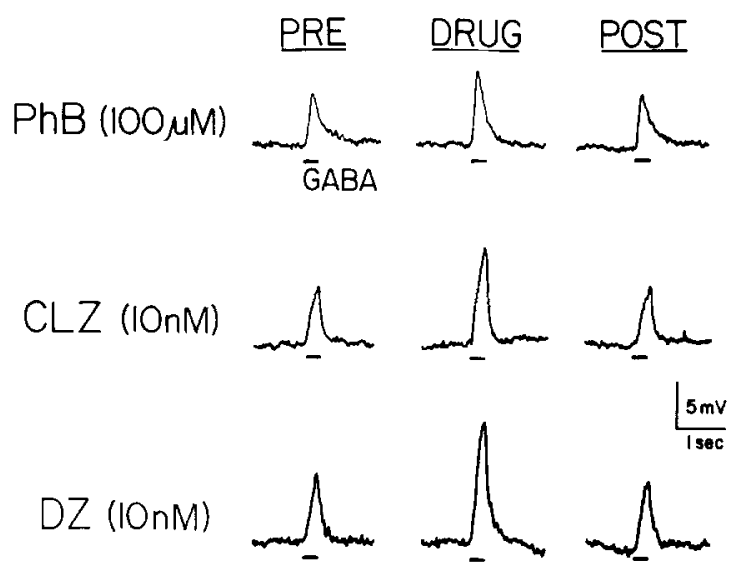

Fig. 3. Barbiturates and benzodiazepines enhanced responses of spinal cord neurons to iontophoretically-applied GABA. Each row shows sequential chart recordings of responses produced in single spinal cord neurons by iontophoretic application of GABA before $(P R E)$, during $(D R U G)$, and after (POST) pressure ejection of antiepileptics from a pressure ejection micropipette position nearby. Membrane potentials were -70 to $-80 \mathrm{mV}$ and intracellular recordings were made using 3 molar $\mathrm{KCl}$ micropipettes. Under these conditions, GABA responses were depolarizing. Phenobarbital, clonazepam, and diazepam rapidly and reversibly enhanced the GABA responses (from Macdonald and MacLean, 1985)

1977; Macdonald and Barker, 1978; Skerritt and Macdonald, 1984) all enhanced iontophoretically produced GABA responses (Fig. 3). Enhancement by all three drugs was rapid in onset and was reversible. Phenobarbital enhanced GABA responses at concentrations above $20 \mu \mathrm{M}$ with a $20-25 \%$ enhancement being produced at $100 \mu \mathrm{M}$. The benzodiazepines clonazepam and diazepam were substantially more potent than phenobarbital, producing enhancements in GABA responses at concentrations above $1 \mathrm{nM}$ and producing $80-100 \%$ enhancements at $100 \mathrm{nM}$. Nitrazepam was slightly less potent than clonazepam and diazepam. Valproic acid and phenytoin enhanced GABA responses only at nontherapeutic concentrations. Carbamazepine, primidone and ethosuximide did not modify GABA responses.

Phenobarbital, but not the benzodiazepines, reduced glutamate responses evoked in spinal cord neurons bathed in a high magnesium solution (Macdonald and Barker, 1979b). The glutamate responses were most likely produced by binding to quisqualate receptors. No attempt to study NMDA or kainate responses was made in these experiments.

\section{Discussion}

We have demonstrated that antiepileptic drugs effective against generalized tonic-clonic seizures and generalized tonic-clonic status epilepticus had an action on neuronal excitability. Phenytoin, carbamazepine and valproic acid blocked sustained high frequency repetitive firing at free serum concentrations that are acieved in ambulatory patients being treated for epilepsy. Phenobarbital and antiepileptic benzodiazepines limited sustained high frequency repetitive firing 
at concentrations achieved during the treatment of generalized tonic-clonic status epilepticus but not at those achieved in ambulatory patients. Finally, ethosuximide did not limit sustained high frequency repetitive firing even at concentrations several fold higher than those achieved in patients being treated for generalized absence seizures. These results suggest that reduction of sustained high frequency repetitive firing by antiepileptic drugs may form, in part, the basis for antiepileptic action against generalized tonic-clonic seizures and generalized tonic-clonic status epilepticus. The mechanism of the limitation of sustained high frequency repetitive firing has not been definitively demonstrated. However, since the antiepileptic drugs have no effect on resting membrane properties of these neurons and since there is a use-dependent and voltagedependent reduction of the maximal rate of rise of action potentials in the trains, it is likely that the antiepileptic drugs are directly affecting sodium channels. The effect of the drugs is not to block directly the channels since single action potentials evoked at all voltages were unaffected by these antiepileptic drugs. The blockade of sodium channels produced by the antiepileptic drugs was use- or frequency-dependent. With repetitive stimulation or during repetitive firing of action potentials within trains, the antiepileptic drugs began to have their effects on maximal rate of rise only after the firing of several action potentials. Furthermore, the reduction in the maximal rate of rise of the action potentials increased as the number of action potentials evoked increased. Block of repetitive firing by the antiepileptic drugs was also voltage-dependent. The blockade could be relieved by membrane hyperpolarization and was exacerbated following membrane depolarization. A common feature of repetitive firing and of membrane depolarization is the increasing presence of inactive sodium channels. These data suggest that the antiepileptic drugs are binding to the sodium channels when they are in their inactive state. This mechanism is similar to the modulated receptor hypothesis that has been used to describe the mechanism of action of local anesthetics (Hille, 1977; Hondeghem and Katzung, 1977). The action of phenytoin on sodium currents has been studied in neuroblastoma cells recently. These studies have confirmed an effect of phenytoin on the inactivation process. However, in one study (Willow et al., 1984), an effect of phenytoin on fast sodium channel inactivation was reported, while in a second study on neuroblastoma neurons (Matsuki et al., 1984), an effect of slow sodium channel inactivation was reported. A major limitation in studying the effect of antiepileptic drugs on sodium currents in vertebrate neurons is their morphology. Vertebrate neurons including those in culture have multiple large dendrites and axons originating from cell bodies or proximal dendrites. Voltage clamp studies are extremely difficult on neurons with such morphology, and it has not been possible for us to study sodium currents in these vertebrate neurons. Therefore, a definitive characterization of the mechanism of antiepileptic drug action on sodium channels may await the application of patch clamp techniques to single sodium channels.

Some antiepileptics drugs also enhance GABA-mediated inhibition. While 
both barbiturates and benzodiazepines enhance GABA responses, their mechanism of action is likely to be different. Benzodiazepines bind to a specific benzodiazepine receptor (Möhler, Okada, 1977; Squires and Braestrup, 1977), while barbiturates appear to enhance GABA responses by binding to the TBPS, or picrotoxin, binding site (Ticku et al., 1978; Squires et al., 1983). Valproic acid did not enhance GABA responses at therapeutic concentrations but has been shown to increase brain GABA levels. It has been suggested that valproic acid increases GABA responses by increasing presynaptic release of GABA. However, this effect of valproic acid has not been demonstrated directly (Godin et al., 1969; see Chapman et al., 1982, for review). Therefore, it remains uncertain whether valproic acid enhances GABAergic synaptic transmission.

The correlation of enhancement of GABAergic synaptic transmission with antiepileptic drug action is less obvious. In experimental animals, the ability of the anticonvulsant drugs to enhance GABA responses correlates well with their ability to suppress pentylenetetrazol-induced clonic seizures. The pentylenetetrazol seizure model is predictive, in general, for actions of antiepileptic drugs on generalized absence seizures. However, while diazepam is a good anti-absence seizure drug, phenobarbital is not. Furthermore, valproic acid and ethosuximide are excellent antiabsence seizure drugs and neither had an action on GABA responses. While it is possible that valproic acid increases GABAergic synaptic transmission by increasing the presynaptic release of transmitter, no direct effect of ethosuximide on a GABA system has been demonstrated (Barnes and Dichter, 1984; McLean and Macdonald, 1986a). Thus, the effect of anti-absence seizure drugs on GABAergic inhibition is poorly correlated. However, the correlation of GABA enhancement with treatment of myoclonic seizures is somewhat better. Phenobarbital, benzodiazepines and valproic acid are all useful drugs in the treatment of myoclonic seizures while ethosuximide is not effective. Thus, if valproic acid does increase the release of GABA, all three antimyoclonic seizure drugs may produce their action through an enhancement of GABAergic synaptic transmission. The question remains, what is the antiabsence seizure mechanism of these antiepileptic drugs. It is possible that the enhancement of GABAergic synaptic transmission is one mechanism whereby antiepileptic drugs can block generalized absence seizures. This, however, cannot be the sole mechanism for antiabsence seizure action. It is almost certain that ethosuximide has a different action than those of the benzodiazepines and valproic acid. This mechanism, however, remains to be elucidated.

\section{References}

Barnes DM, Dichter MA (1984) Effects of ethosuximide and tetramethyl-succinimide on cultured cortical neurons. Neurology 34: 620-625

Chapman A, Keane PE, Meldrum BS, Simiand J, Vernieres JC (1982) Mechanisms of anticonvulsant action of valproate. Prog Neurobiol 19: 315-359

Choi D, Farb D, Fischbach B (1977) Chlordiazepoxide selectively augments GABA action in spinal cord cell cultures. Nature 269: 342-344 
Godin Y, Heiner L, Mark J, Mandel P (1969) Effects of di-n-propylacetate, an anticonvulsant compound, on GABA metabolism. J Neurochem 19: 869-873

Hille B (1977) Local anesthetics: hydropholic and hydrophobic pathways for the drugreceptor reaction. J Gen Physiol 69: 497-515

Hodeghem LM, Katzung BB (1977) Time- and voltage-dependent interactions of antiarrhythmic drugs with cardiac sodium channels. Biochim Biophys Acta 472: 373-398

Krall RL, Penry JK, White BG, Kupferberg HJ, Swinyard EA (1978) Antiepileptic drug development: II. Anticonvulsant drug screening. Epilepsia 19: 409-428

Macdonald RL (1983) Mechanisms of anticonvulsant drug action. In: Pedley TA, Meldrum B (eds) Recent advances in epilepsy, vol 1. Churchill, Livingstone, Edinburgh, pp 123

Macdonald RL, Barker JL (1978) Benzodiazepines specifically modulate GABA-mediated post-synaptic inhibition in cultured mammalian neurons. Nature 271: 563-564

Macdonald RL, Barker JL (1979a) Enhancement of GABA-mediated postsynaptic inhibition in cultured mammalian neurons: a common mode of anticonvulsant action. Brain Res 167: 323-336

Macdonald RL, Barker JL (1979b) Anticonvuisant and anesthetic barbiturates: different postsynaptic actions in cultured mammalian neurons. Neurology 29: 432-447

Macdonald RL, McLean MJ, Skerritt JH (1985) Anticonvulsant drug mechanisms of action. Fed Proc 44: 227-235

Macdonald RL, McLean MJ (1986) Anticonvulsant drugs: mechanisms of action. In: Delgado-Esqueta AV, Ward Jr AA, Woodbury DM, Porter RJ (eds) Basic mechanisms of the epilepsies, vol 44. Raven Press, New York, pp 713-736

Matsuki N, Quandt FN, Ten Eick RE, Yeh JZ (1984) Characterization of the block of sodium channels by phenytoin in mouse neuroblastoma cells. J Pharmacol Exp Ther 228: $523-530$

McLean MJ, Macdonald RL (1983a) Phenytoin and carbamazepine selectively limit sustained high frequency repetitive firing of cultured mouse neurons. Soc Neurosci Abst 9: 398

McLean MJ, Macdonald RL (1983b) Multiple actions of phenytoin on mouse spinal cord neurons in cell culture. J Pharmacol Exp Ther 227: 779-789

McLean MJ, Macdonald RL (1986 a) Sodium valproate, but not ethosuximide, produces use- and voltage-dependent limitation of high frequency repetitive firing of action potentials of mouse central neurons in cell culture. J Pharmacol Exp Ther 237: 10011011

McLean MJ, Macdonald RL (1986 b) Carbamazepine and 10,11-epoxy carbamazepine produced use- and voltage-dependent limitation of rapidly firing action potentials of mouse central neurons in cell cultures. J Pharmacol Exp Ther 238: 727-732

Möhler H, Okada T (1977) Demonstration of benzodiazepine receptors in the central nervous system. Science 198: 849-851

Nau H, Löscher W (1982) Valproic acid: brain and plasma levels of the drug and its metabolites, anticonvulsant effects and $\gamma$-aminobutyric acid (GABA) metabolism in the moue. J Pharmacol Exp Ther 220: 654-659

Schulz DW, Macdonald RL (1981) Barbiturate enhancement of GABA-mediated inhibition and activation of chloride ion conductance: correlation with anticonvulsant and anesthetic actions. Brain Res 209: 177-188

Skerritt JH, Macdonald RL (1984) Benzodiazepine receptor ligand actions on GABA responses: benzodiazepines, CL 218872, zopiclone. Eur J Pharmacol 101: 127-134

Skerritt JH, Rock DM, McLean MJ, Macdonald RL (1984a) Concentration-dependent effects of benzodiazepines on GABA responses and sustained high frequency repetitive firing in mouse cultured neurons. Soc Neurosci Abstr 10:632

Skerritt JH, Werz MA, McLean MJ, Macdonald RL (1984b) Diazepam and its anomalous 
p-chloro-derivative Ro 5-4864: comparative effects on mouse neurons in cell culture. Brain Res 310: 99-105

Squires RF, Braestrup C (1977) Benzodiazepine receptors in rat brain. Nature 266: 732734

Squires RF, Casida JE, Richardson M, Saederup E (1982) $\left[{ }^{35}\right.$ S]t-butylbicy-clophosphorothionate binds with high affinity to brain-specific sites coupled to $\gamma$-aminobutyric acid-A and ion recognition sites. Mol Pharmacol 23: 326-336

Ticku MK, Ban M, Olsen RW (1978) Binding of $\left[{ }^{3} \mathrm{H}\right] \alpha$-dihydropicrotoxinin, a $\gamma$-aminobutyric acid synaptic antagonist, to rat brain membranes. Mol Pharmacol 14: 391-402

Willow M, Kuenzel EA, Catterall WA (1983) Inhibition of voltage, sensitive sodium channels in neuroblastoma cells and synaptosomes by the anticonvulsant drugs diphenylhydantoin and carbamazepine. Mol Pharmacol 25: 228-234

Woodbury DM, Penry JK, Pippenger CE (1982) Antiepileptic drugs, 2nd edn. Raven Press, New York

Author's address: Dr. R. L. Macdonald, 1103 East Huron, Ann Arbor, MI 48104, U.S.A. 